\title{
Rêve fantômes fantasmes, «Ponts/Ponti» 14
}

\section{Carminella Biondi}

\section{(2) OpenEdition}

\section{Journals}

\section{Edizione digitale}

URL: http://journals.openedition.org/studifrancesi/4493

DOI: $10.4000 /$ studifrancesi.4493

ISSN: 2427-5856

\section{Editore}

Rosenberg \& Sellier

\section{Edizione cartacea}

Data di pubblicazione: 1 settembre 2016

Paginazione: 372-373

ISSN: 0039-2944

\section{Notizia bibliografica digitale}

Carminella Biondi, «Rêve fantômes fantasmes, «Ponts/Ponti» 14 », Studi Francesi [Online], 179 (LX | II) |

2016, online dal 01 septembre 2016, consultato il 18 septembre 2020. URL : http://

journals.openedition.org/studifrancesi/4493; DOI : https://doi.org/10.4000/studifrancesi.4493

\section{Questo documento è stato generato automaticamente il 18 settembre 2020.}

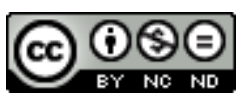

Studi Francesi è distribuita con Licenza Creative Commons Attribuzione - Non commerciale - Non opere derivate 4.0 Internazionale. 


\title{
Rêve fantômes fantasmes, «Ponts/ Ponti» 14
}

\author{
Carminella Biondi
}

\section{NOTIZIA}

Rêve fantômes fantasmes, «Ponts/Ponti» 14, 2015, 350 pp.

Questo numero, dedicato alla presenza e alla scrittura dell'onirico nelle letterature francofone, indica fin dalla scelta del titolo, che scandisce le diverse, possibili declinazioni del sogno, la volontà di affrontare l'ampio spettro che la tematica propone. Anche se, inevitabilmente, il risultato è più simile a una serie di carotaggi, destinati a portare in superficie materiali preziosi, che a una panoramica organica dell'argomento, impossibile da realizzarsi visto che gli articoli sono soltanto sei, su tempi e spazi molto dilatati: si va infatti dalla Nouvelle-France del Seicento, nella lettura della Relation del 1654 di Marie de l'Incarnation, al Belgio di Robert Poulet, dall'Algeria, al Libano, al Cameroun, e che la breve introduzione del nuovo direttore, Marco Modenesi, non tenta neppure, giustamente, una sintesi problematica di un soggetto tanto vasto, analizzato in tempi e spazi altrettanto vasti. Al lettore l'arduo compito.

2 Ciò non toglie merito ai singoli lavori qui proposti e al loro risultato d'insieme: un apporto di conoscenze preziose e inedite su autori noti e meno noti e un'apertura su prospettive di ricerca potenzialmente molto feconde. Per esempio, il primo articolo di Éléonore Julie QUINAUX (De "Handji" aux "Ténèbres" di Robert Poulet, pp. 11-22), ci introduce alla conoscenza del meno noto fratello del famoso critico Georges Poulet e alle caratteristiche e alla funzione originale del sogno nella sua opera narrativa: «Robert Poulet mène ses lecteurs du réél initial vers un réel rêvé et d'un réél rêvé vers une recontextualisation mystique. Dans cette projection mystique, l'individu n'y a plus de place, seul la masse compte» (p. 20). Un ruolo davvero inedito dell'onirico nella scrittura narrativa, che ha la forza di coinvolgere il lettore in una nuova visione del mondo. 
3 Il secondo articolo, opera di uno studioso molto noto della scrittura giudaicomaghrebina di lingua francese, Guy DUGAS (La "Eubéita" et autres fantasmes animaliers chez les écrivains francophones judéo-tunisiens francophones de l'entre-deux-guerres, pp. 23-32), analizza la presenza ossessiva dei fantasmi animali nel ghetto, e in particolare del fantasma indicato nel titolo, nei racconti di alcuni intellettuali ebrei che hanno dato vita alla cosiddetta «École de Tunis». Si passa dalla Tunisia all'Algeria nell'articolo che segue di Ines BURGET: Un auteur algérien en dialogue avec les fantômes littéraires: "L'Imposture des mots" de Yasmina Khadra, pp. 33-59). Come tutti sappiamo, il nome dell'autore è uno pseudonimo dietro il quale si è nascosto per anni l'ufficiale Mohammed Moulessehoul che, nel 2001, nell'Écrivain, ha deciso finalmente di rivelare la sua identità, non senza sollevare problemi presso i suoi lettori, ma soprattutto di ordine personale, identitario, come indica, nel testo qui preso in esame, il dialogo instaurato dall'autore Yasmina Khadra con l'ufficiale-scrittore che ne ha indossato la maschera, ma anche con i suoi (loro?) personaggi, che come capita spesso nella letteratura contemporanea, in particolare nella letteratura postcoloniale, reclamano la propria autonomia e infine il dialogo fra lo scrittore (nella sua duplice veste) e quelli che ha considerato i suoi maestri, che possono essere, indifferentemente, autori o personaggi (vedi il caso di uno Zarathustra decaduto, divenuto barbone in una strada di Parigi). Il tutto in una sarabanda schizofrenica che pone in maniera tangibile il problema della complessità inestricabile dello statuto dello scrittore, di quello del lettore e della funzione della scrittura in un mondo quanto mai instabile.

Con Simonetta VALENTI si passa in Libano (Fantômes et fantasmes de l'histoire dans "Anima" de Wajdi Mouawad, pp. 61-67), attraverso l'analisi approfondita di un romanzo dello scrittore libano-quebecchese Wajdi Mouawad, già molto noto come autore drammatico. La tematica non cambia rispetto alle opere di teatro: le guerre e la martoriata situazione della terra d'origine, ma il genere permette un'analisi più approfondita degli stati d'animo che accompagnano la «quête» a ritroso e le scoperte sempre più drammatiche e destabilizzanti del giovane protagonista, Wahhch Debch, che scopre, insieme al significato sinistro del suo nome (Monstrueux Brutal), di essere stato allevato non da un infermiere-benefattore, dopo la perdita della sua famiglia, come aveva sempre creduto, ma dall'assassino-torturatore che lo ha reso orfano. La brutalità della guerra e delle sue bestiali conseguenze emerge qui in tutta la sua forza distruttiva, una forza che continua nel tempo: «les victimes d'antan sont devenues les bourreaux d'aujourd'hui et les tortionnaires d'autrefois se changent soudain en martyrs...» (p. 74). Bisogna spezzare il filo, parlare una lingua nuova, cercare una coabitazione. Ma è veramente possibile? L'articolo che segue, di Jada MICoNI, indaga Le rêve dans "Le dernier gardien de l'arbre" de Jean-Roger Essomba (pp. 80-93), un autore di origine camerunense, che, come molti scrittori africani, vive attualmente in Francia. Qui ci troviamo di fronte a sogni reali dei protagonisti a forte connotazione profetica, al punto da condizionarne l'esistenza. Il passato, come si scoprirà nel corso del romanzo, accomuna i due protagonisti, provenienti da storie e paesi diversi (il Cameroun, dove si svolge l'azione, e la Francia). La tradizione vuole che il vecchio albero, simbolo di tutto un popolo, abbia sempre un "gardien», che, come accadeva alle vestali dei tempi antichi, deve sacrificare la propria natura di uomo attraverso la castrazione. Ma non sarà il predestinato, bensì un prete cattolico venuto in Africa per scontare, come gli ha rivelato un sogno, il suo passato di capitano negriero ad assumersi l'importante e pesante impegno, in 
un'unione simbolica tra un passato di distruzione e di morte e un presente in cui una riconciliazione è possibile.

Il dossier si conclude con un salto indietro nel tempo, all'epoca della prima colonizzazione del Canada, o meglio della Nouvelle-France, attraverso l'analisi puntuale di due sogni profetici, inseriti nella Relation del 1654, di Marie de l'Incarnation, una donna straordinaria che ha fondato al di là dell'Atlantico il primo convento delle Orsoline (Les rêves prophétiques de Marie de l'Incarnation. 1599-1672, pp. 95-114). Anche qui, come nel saggio precedente, ci troviamo di fronte a sogni che orientano il percorso di una vita, prima verso la scelta monacale, nonostante questo comporti l'abbandono di un figlio, poi verso la scelta missionaria. Amandine Bonesso analizza bene la scrittura di questi sogni, certamente veritiera, ma anche funzionale alla giustificazione di certe difficili scelte esistenziali e all'esaltazione del valore di una vita tracciata dall'alto.

6 Seguono, fuori dossier, un saggio di carattere linguistico sui neologismi in un giornale satirico camerunense: La néologie dans "Le Messager Popoli": reflet du visage sociopolitique camerounais de 1993 à 2010 (pp. 117-133) di Cécile Madiga, e le ricche pagine di rassegna critica (pp. 137-240), che costituiscono uno dei punti di forza della rivista. 